\title{
MHz Bandwidth Electro-optical Modulator based on a Reconfigurable Photonic Metamaterial
}

\author{
J. Y. Ou, E. Plum, and N. I. Zheludev \\ Optoelectronics Research Centre and Centre for Photonic Metamaterials \\ University of Southampton, Southampton SO17 1BJ, United Kingdom
}

\begin{abstract}
We provide the first demonstration of megahertz-rate electro-optical modulation using a reconfigurable photonic metamaterial driven by electrostatic forces. The modulator can also be engaged in nonvolatile switching providing $90 \%$ switching contrast.

OCIS codes: (160.3918) Metamaterials; (250.5403) Plasmonics
\end{abstract}

We developed a nano-electro-mechanical switchable (NEMS) photonic metamaterial that provides a flexible and convenient platform for megahertz rate modulation in the optical and near-infrared parts of the spectrum. Its high frequency response, low switching power and high-contrast modulation are unique advantages of this new technology.

The nano-electro-mechanical switchable reconfigurable photonic metamaterial reported here is driven by electrostatic forces. The electro-optical metamaterial device comprises of a gold plasmonic nano-wire pattern fabricated on a dielectric membrane (Fig.1). It operates in the optical telecom range of wavelengths and can be used as $\mathrm{MHz}$ bandwidth modulator consuming only a few microwatts of power. It can also be engaged in a non-volatile switching providing more that 90 percent transmission change upon switching (Fig.2).
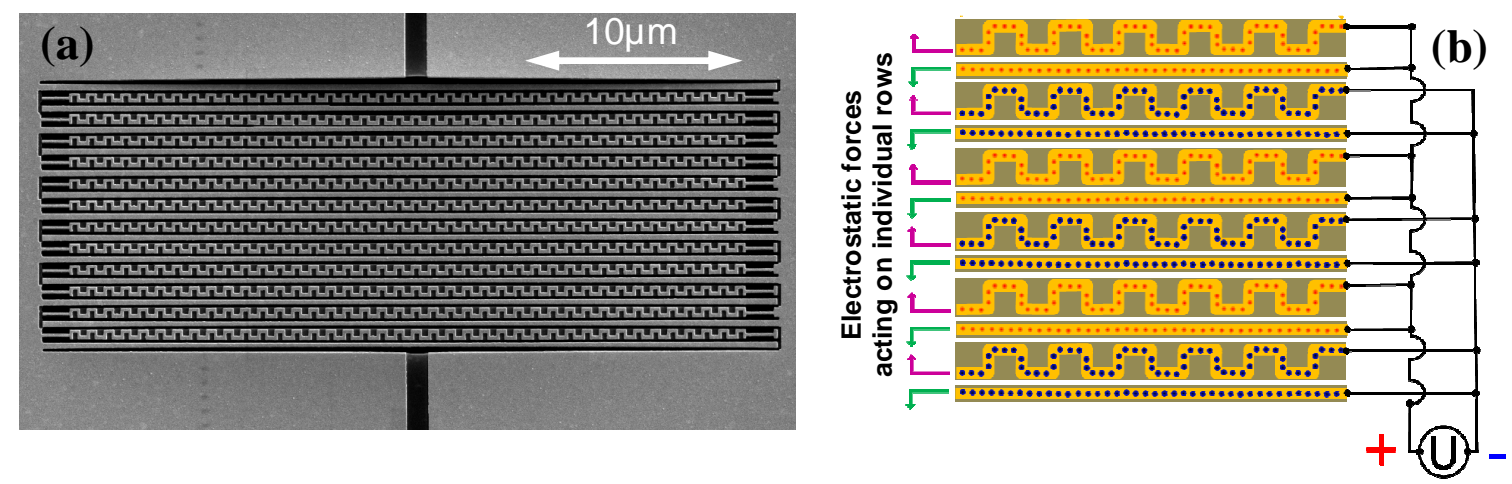

(c)

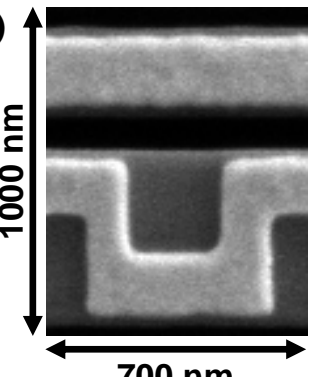

(d)

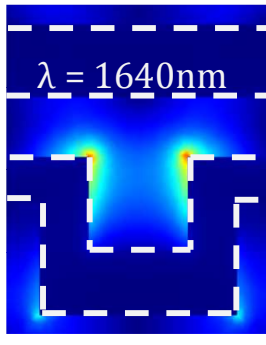

off

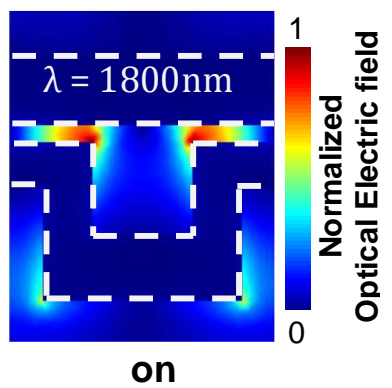

on

Fig. 1. Nano-electro-mechanical switchable (NEMS) photonic metamaterial. (a) Scanning electron microscope image of the entire device showing the gold metal nanostructure supported by flexible bridges cut from a SiN membrane. (b) Schematic of a section of the metamaterial pattern and the driving circuit. (c) Detail of a single unit cell. (d) Resonant modes of excitation for the devices's "off" and "on" states.

The metamaterial was manufactured by focused ion beam milling on a $50 \mathrm{~nm}$ thick silicon nitride membrane that provides a stable and flexible base for the metallic pattern. The metallic pattern, which is predominantly responsible for resonant optical properties of the whole structure, consists of a meander design alternating with straight metal wires. The meander pattern provides a resonant response linked to the excitation of a plasmonic mode (Fig. 1d). The variable distance between the straight wire and the meander-shaped conductor controls the resonant optical response 
of the nanostructure. Control over this spacing is achieved by manufacturing the $50 \mathrm{~nm}$ thick straight and meander gold wires on different flexible bridges that were cut from the silicon nitride membrane. The $500 \mathrm{~nm}$ and $230 \mathrm{~nm}$ wide bridges were separated by $125 \mathrm{~nm}$ gaps to provide room for relative motion and the ends of the bridges were narrowed to increase flexibility as shown on the SEM image. In order to make the bridge spacing adjustable, pairs of bridges were alternatingly connected to two electrical terminals of the device, which allow the electrostatic forces between the bridges to be controlled (Fig. 1b). The section of the metamaterial pattern that was available for optical modulation was $12 \times 25$ microns in size.

(a)

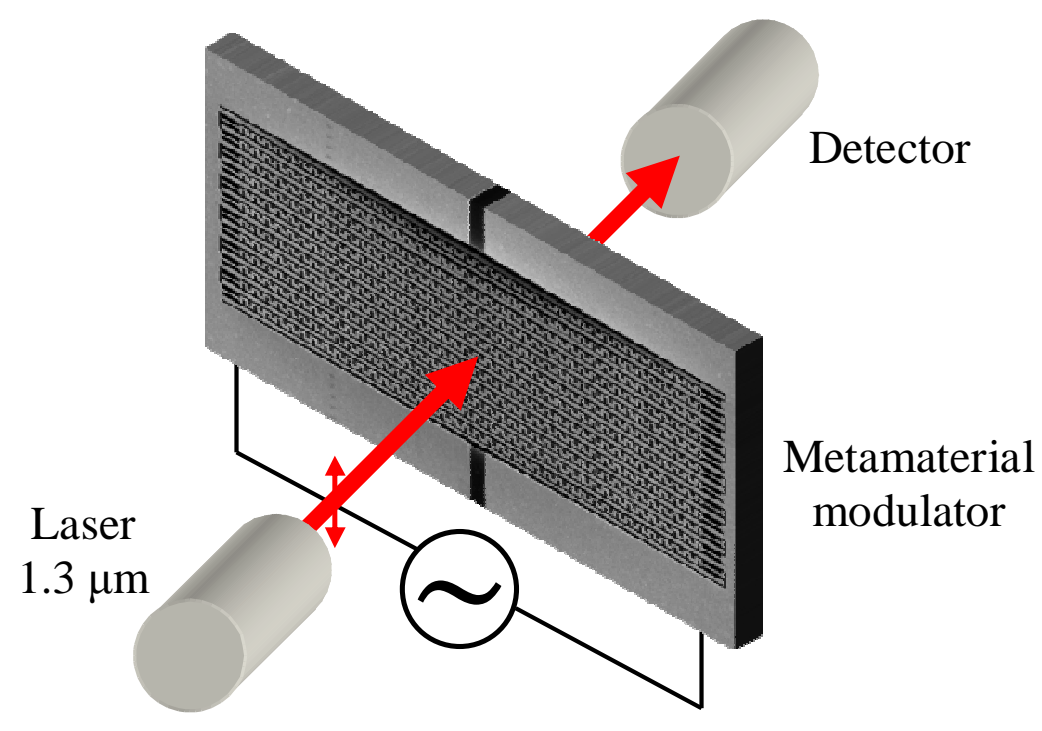

(b)

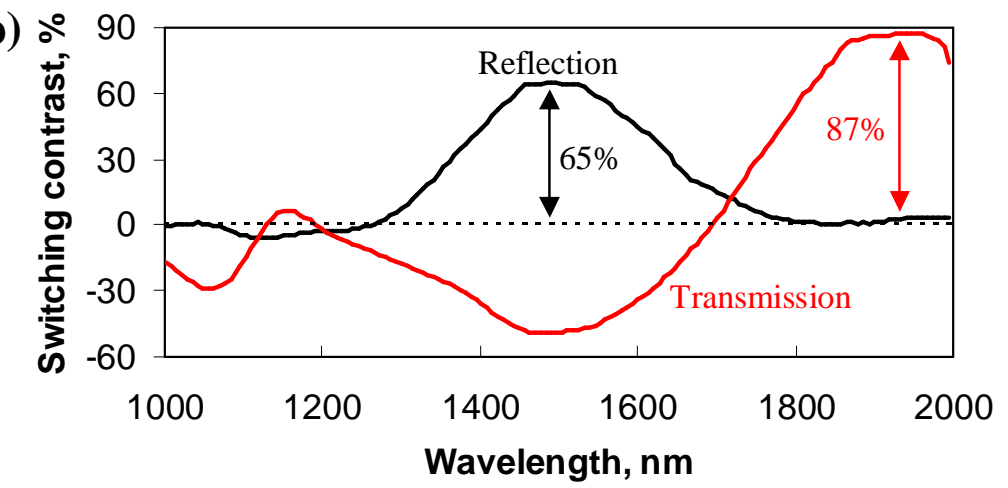

(c)

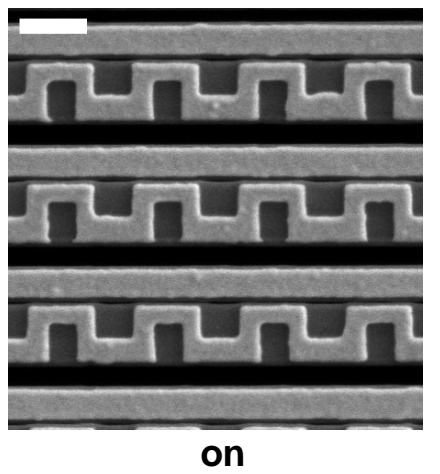

Fig. 2. Electro-optic modulator and switch. (a) Schematic of the electro-optical modulator. (b) Switching contrast between the metamaterial's "on" and "off" states in terms of relative transmission and reflection changes, $\Delta \mathrm{T} / \mathrm{T}_{\text {off }}$ and $\Delta \mathrm{R} / \mathrm{R}_{\text {off. }}$ (c) Scanning electron microscope image of the metamaterial in its switched "on" state.

For applied voltages of up to $\sim 6 \mathrm{~V}$, the spacing between the bridges can be continuously controlled as the restoring force of the elastic structure can balance the electrostatic forces between the bridges. This allows continuous tuning and modulation of the metamaterial properties. Measurements of the metamaterial's transmission at a wavelength of $1.3 \mu \mathrm{m}$ show that its optical properties can be controlled with modulation frequencies of up to about $10 \mathrm{MHz}$.

Non-volatile switching occurs at $\sim 6 \mathrm{~V}$, when the electrostatic force overcomes the elastic restoring force of the silicon nitride bridges. Here, the structure switches into a bridge-pair configuration (Fig. 2c), leading to a dramatic $20 \%$ red-shift of the metamaterial's spectral response and relative transmission changes of up to $90 \%$ (Fig. 2b).

In summary, we demonstrate fast electro-optical modulation and high-contrast switching with an electrostatically actuated NEMS photonic metamaterial that has megahertz bandwidth. 